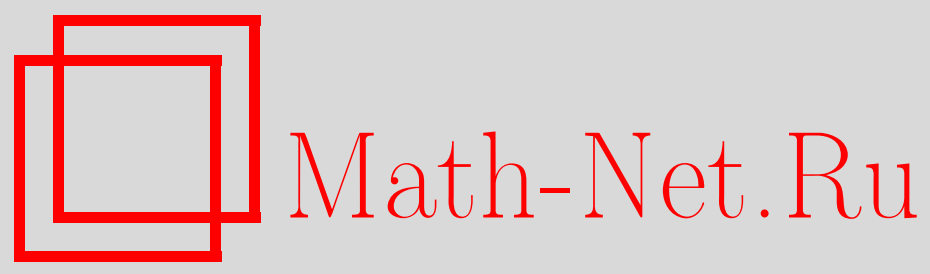

Б. В. Карпов, Алгебро-геометрические аспекты $S$-двойственности, Матем. заметки, 1997, том 61, выпуск 2, 163-178

DOI: https://doi.org/10.4213/mzm1492

Использование Общероссийского математического портала Math-Net.Ru подразумевает, что вы прочитали и согласны с пользовательским соглашением http://www . mathnet.ru/rus/agreement

Параметры загрузки:

IP : 54.157 .27 .8

26 апреля 2023 г., $17: 03: 23$

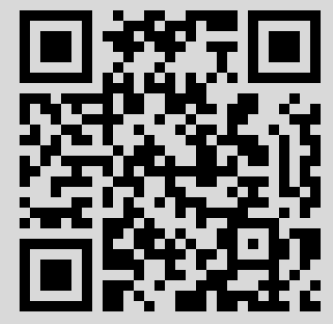




\title{
АЛГЕБРО-ГЕОМЕТРИЧЕСКИЕ АСПЕКТЫ $S$-ДВОЙСТВЕННОСТИ
}

\author{
Б. В. Карпов
}

В работе рассматриваются алгебро-геометрические задачи, связанные с проверкой гипотезы $S$-двойственности для суперсимметричных янг-миллсовских квантовых теорий поля в четырехмерном случае. Описываются все случаи равенства нулю и отрицательности бета-функции Гелл-Мана-Лоу для рангов 1 и 2 калибровочной группы и указываются некоторые серии таких случаев для произвольного ранга калибровочной группы. При реализации одной из серий на комплексной проективной плоскости обнаруживается связь с исключительными расслоениями.

Библиографой: 15 названий.

\section{Введение}

Важной и интересной задачей геометрии является проверка гипотезы $S$-двойственности для суперсимметричных янг-миллсовских квантовых теорий поля в четырехмерном случае (как правило, работают со случаем SYM, $N=4$ или $N=2+$ массивный гипермультиплет в присоединенном представлении с некоторым количеством $N_{f}$ ароматов частиц). Напомним, что для того, чтобы можно было вычислять квантовые коррелящионные функции в квазиклассическом пределе, нужно, чтобы бета-функция Гелл-Мана-Лоу полей этой теории была равна нулю (или в крайнем случае была бы отрицательной). В этом случае корректно определена константа калибровочного взаимодействия (либо электромагнитного - $e$, либо сильного - $g$ ). Кроме того, поскольку бета-функция равна нулю, не существует аномалий $U(1)$-глобальной симметрии, т.е. физика зависит от вакуумного угла $\theta$, и наша теория, конечно, масштабно инвариантна. Таким образом, как параметр нашей теории возникает константа

$$
\tau=\frac{\theta}{\pi}+\frac{8 \pi i}{g^{2}}
$$

(принадлежащая верхней полуплоскости) и гипотеза $S$-двойственности заключается в том, что статсумма нашей теории $Z_{\tau}$ ведет себя как модулярная форма при целочисленных дробно-линейных преобразованиях (предположения об этой симметрии были сделаны Монтаненом и Оливом более двадцати лет назад [1]). Для проверки этого чисто

Работа выполнена при частичной финансовой поддержке Российского фонда фундаментальных исследований, грант № 96-01-01323. 
математического факта Вафа и Виттен [2] предложили метод, который, к сожалению, относится только к частным случаям четырехмерных многообразий (алгебраических поверхностей дель Пещо и типа К3), для которых справедливы теоремы зануления гармонических спиноров в каноническом или присоединенном представлениях.

Цель настоящей работы - описать все случаи, когда вышеупомянутые методы применимы для небольших рангов (1 и 2) калибровочной групшы и указать некоторые серии таких случаев для произвольного ранга калибровочной групшы. В частности, мы показываем, что полная программа проверки интересующей физиков $S$-двойственности при “больших энергиях" для рангов 1 и 2 калибровочной группы сводится к пяти случаям (математические исследования в которых уже ведутся), а для произвольного ранга калибровочной группы представлены три серии, над двумя из которых работают физики (для серии а) см. [2] и для серии б) см. [3]).

Необходимо заметить, что существует параллельная программа проверки $S$-двойственности при "малых энергиях", связанная с чисто геометрическим описанием динамики кулоновской ветви вакуума [3], [4]. Этого подхода мы здесь не касаемся.

Автор благодарен А. Н. Тюрину за многочисленные полезные обсуждения, без которых написание этой статьи было бы невозможно, а также всем участникам семинара по алгебре в Математическом институте им. В. А. Стеклова РАН за внимание и интерес к данной работе.

Основной отсев квантовых теорий поля, годных для тестирования высокоэнергетической $S$-двойственности, дает условие на бета-функцию. С этого мы и начнем.

\section{$\S 1$. Бета-функция}

Математическое содержание этого параграффа, конечно, хорошо знакомо экспертам по теории представлений, но мы приводим его лишь для удобства перехода к геометрии.

1.1. Пусть $\gamma$ - неприводимое конечномерное комплексное представление алгебры Ли $\mathrm{su}(r)$ (алгебры Ли калибровочной группы нашей теории) и $b_{\gamma}$ - элемент Казимира этого представления. Обозначим через $c_{2}(\gamma)$ коэффициент пропорциональности между $b_{\text {can }}$ и $b_{\gamma}$ :

$$
b_{\text {can }}=c_{2}(\gamma) b_{\gamma}
$$

где сап - каноническое представление $\mathrm{su}(r)$ в $\mathbb{C}^{r}$.

Рассмотрим конечный набор представлений $\left\{\gamma_{1}, \ldots, \gamma_{N_{a}}\right\}$, среди которых есть эквивалентные. Поднабор, состоящий из попарно эквивалентных представлений, соответствует частице, число представлений в нем - число $N_{f}$ ароматов частицы, а размерность $r$ канонического представления калибровочной групш - число цветов. Таким образом, $N_{a}$ есть общее число ароматов частиц нашего ансамбля. Бета-функция набора $\left\{\gamma_{1}, \ldots, \gamma_{N_{a}}\right\}$ имеет вид

$$
\beta\left(\left\{\gamma_{i}\right\}\right)=-c_{2}(\mathrm{ad})+\sum_{i} c_{2}\left(\gamma_{i}\right)
$$

ЗАмЕчАниЕ. Хотя обозначение $c_{2}(\gamma)$ не совсем традиционно в теории алгебр Ли, оно себя оправдывает при последующем переходе к геометрии. А именно, представление $\gamma$ можно рассматривать как тензорную операцию на расслоениях ранга $r$, и $c_{2}(\gamma)$ есть коэффициент при $c_{2}(E)$ в выражении $c_{2}(\gamma(E))$ через классы Чжэня расслоения $E$. 
Цель этого параграфа - описать все случаи, когда $\beta\left(\left\{\gamma_{i}\right\}\right) \leqslant 0$ для алгебр su(2) и $\mathrm{su}(3)$, и указать некоторые серии таких случаев для алгебр $\mathrm{su}(r)$.

ЗАмЕчАниЕ. Поскольку мы работаем с алгебрами Ли, у нас остается еще свобода в выборе калибровочной групшы. Например, $\mathrm{su}(2) \cong \mathrm{so}(3)$, что соответствует выбору детерминанта расслоения по модулю ранга.

Любое комплексное представление алгебры su( $r)$ продолжается по линейности на ее комплексификацию $\mathrm{sl}(r, \mathbb{C})$ без изменения $c_{2}$, поэтому мы будем рассматривать неприводимые конечномерные представления $\operatorname{sl}(r, \mathbb{C})$. Пусть $\gamma-$ такое представление и $\left\{e_{i}\right\}-$ базис в $\operatorname{sl}(r, \mathbb{C})$. Рассмотрим двойственньй базис $\left\{f_{i}\right\}$ относительно билинейной формы

$$
B_{\gamma}(X ; Y)=\operatorname{Tr}(\gamma(X) \gamma(Y))
$$

т.е. такой, что $B_{\gamma}\left(e_{i} ; f_{j}\right)=\delta_{i j}$. По определению

$$
b_{\gamma}=\sum_{i} e_{i} f_{i}
$$

Это центральный элемент универсальной обертьвающей алгебры, он не зависит от выбора базиса.

Поскольку для двойственного представления $B_{\gamma^{*}}(X ; Y)=B_{\gamma}(X ; Y)$, то справедливо равенство

$$
c_{2}\left(\gamma^{*}\right)=c_{2}(\gamma)
$$

Мы можем указать два подхода к вычислению коэффициентов пропорциональности между элементами Казимира. В основе первого из них лежит следующее утверждение.

ПРЕДЛОЖЕНИЕ 1. Все билинейные формы $B_{\gamma}$ для конечномерных представлений алгебры $\mathrm{sl}(r, \mathbb{C})$ пропорциональны.

СлЕДСТВИЕ. $B_{\gamma}=c_{2}(\gamma) B_{\text {can }}$.

Доказательство предложения мы проводим отдельно для $\operatorname{sl}(2, \mathbb{C})$ (ранг 1 ) и в общем случае. После этого задача сводится к вычислению или оценке ненулевого матричного элемента билинейной формы $B_{\gamma}$ в стандартном базисе $\operatorname{sl}(r, \mathbb{C})$.

Второй подход приводит к общей формуле, выражающей $c_{2}(\gamma)$ через старший вес представления $\gamma$. Этот подход основьвается на двух формулах теории представлений полупростых алгебр Ли. Первая из них - формула Фрейденталя, примененная к старшему весу (см., например, [5, формула (25.14)]), которая состоит в том, что элемент

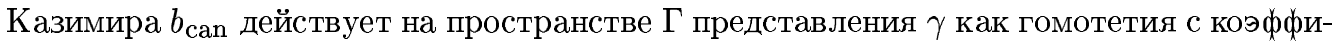
циентом

$$
(\lambda ; \lambda+2 \rho)=\|\lambda+\rho\|^{2}-\|\rho\|^{2} .
$$

Здесь $\lambda$ - старший вес представления $\gamma$ относительно некоторого базиса системы корней, $\rho$ - полусумма положительных корней и $(\cdot ; \cdot)$ - форма Киллинга. Из формулы Фейденталя вытекает, что

$$
\operatorname{Tr}\left(\gamma\left(b_{\text {can }}\right)\right)=c_{2}(\gamma) \operatorname{Tr}\left(\gamma\left(b_{\gamma}\right)\right)=(\lambda ; \lambda+2 \rho) \operatorname{dim} \Gamma
$$


С другой стороны, из определения $b_{\gamma}$ имеем

$$
\operatorname{Tr}\left(\gamma\left(b_{\gamma}\right)\right)=\sum_{i} \operatorname{Tr}\left(\gamma\left(e_{i}\right) \gamma\left(f_{i}\right)\right)=\sum_{i} 1=\operatorname{dimsl}(r, \mathbb{C})=r^{2}-1
$$

Окончательно получаем

$$
c_{2}(\gamma)=\frac{(\lambda ; \lambda+2 \rho)}{r^{2}-1} \operatorname{dim} \Gamma=\frac{(\lambda ; \lambda+2 \rho)}{r^{2}-1} \prod_{\alpha \in R_{+}} \frac{(\lambda+\rho ; \alpha)}{(\rho ; \alpha)}
$$

Последнее равенство справедливо в силу формулы для размерности пространства представления с данным старшим весом [5, формула (24.6)], и это есть вторая формула теории представлений, на которой основано вычисление $c_{2}(\gamma)$. В общем случае использование формулы (3) затруднено в силу громоздкости произведения по положительным корням, равного $\operatorname{dim} \Gamma$, однако, для симметрических и внешних степеней канонического представления $c_{2}$ легко вычисляется (см. (7)).

В качестве ответа получаем следующее утверждение.

ТЕОРема 1. 1. Для алгебры $\mathrm{su}(2) \quad \beta\left(\left\{\gamma_{i}\right\}\right)=0$ в следующих случаях:

a) $N_{a}=1 u \gamma_{1}=\mathrm{ad}$

б) все $\gamma_{i}=$ can $u N_{a}=4$.

Неравенство $\beta\left(\left\{\gamma_{i}\right\}\right)<0$ мохет иметь место только в случае б) при $N_{a}<4$.

2. Для алгебры $\mathrm{su}(3) \quad \beta\left(\left\{\gamma_{i}\right\}\right)=0$ в следующих случаях:

a) $N_{a}=1 u \gamma_{1}=\mathrm{ad}$;

б) все $\gamma_{i}$ изоморфны либо сап, либо сап $\cong \Lambda^{2}(\mathrm{can}), u N_{a}=6$;

в) $N_{a}=2$, одно из $\gamma_{i}$ изоморфно либо сап $\cong \Lambda^{2}\left(\mathrm{can}^{*}\right)$, либо сап* $\cong \Lambda^{2}(\mathrm{can}), a$ другое - либо $S^{2}(\mathrm{can})$, либо $S^{2}\left(\mathrm{can}^{*}\right)$.

Неравенство $\beta\left(\left\{\gamma_{i}\right\}\right)<0$ может иметь место или в случае б) при $N_{a}<6$, или в случае одного представления, изоморфного либо $S^{2}(\mathrm{can})$, либо $S^{2}\left(\mathrm{can}^{*}\right)$.

3. Для алгебр $\mathrm{su}(r), r \geqslant 2$, имеет место по менъшей мере три серии случаев, в которых $\beta\left(\left\{\gamma_{i}\right\}\right)=0$ :

a) $N_{a}=1 u \gamma_{1}=\mathrm{ad}$;

б) все $\gamma_{i}$ изоморфны либо сап, либо сап $\cong \Lambda^{r-1}(\operatorname{can}), u N_{a}=2 r$;

в) $N_{a}=2$, одно из $\gamma_{i}$ изоморфно либо $\Lambda^{2}(\mathrm{can})$, либо $\Lambda^{2}\left(\mathrm{can}^{*}\right)$, а другое - либо $S^{2}(\mathrm{can})$, либо $S^{2}\left(\mathrm{can}^{*}\right)$.

Для алгебр su(2) и su(3) в некоторых из этих случаев алгебро-геометрическая задача угадана заранее, и соответствующие вычисления ведутся с использованием метода Вафа-Виттена (геометрическая ситуация зануления). Например, случай 1а) исследован в [2], случай 1б) - в [6], алгебро-геометрическая задача для случая 2б) рассмотрена в [7]. Случай 2а) геометрически аналогичен случаю 1a) (см. ниже). Наконец, самьй интересный случай 2в) является единственной реализацией серии 3 в) на поверхностях дель Пещо, ему посвящен последний параграф этой работы. 
1.2. Доказательства утверждений для $\mathrm{su}(2)$. Стандартный базис алгебры $\mathrm{sl}(2, \mathbb{C})$ имеет вид

$$
H_{0}=\left(\begin{array}{cc}
1 & 0 \\
0 & -1
\end{array}\right), \quad X_{0}=\left(\begin{array}{ll}
0 & 1 \\
0 & 0
\end{array}\right), \quad Y_{0}=\left(\begin{array}{ll}
0 & 0 \\
1 & 0
\end{array}\right)
$$

Известно [5], что любое конечномерное неприводимое представление $\mathrm{sl}(2, \mathbb{C}$ ) (отличное от тривиального одномерного) изоморфно $\gamma_{n}=S^{n}(\operatorname{can})$ для некоторого $n \geqslant 1$. При этом в пространстве представления можно выбрать базис $\left\{\xi_{k}\right\}, 0 \leqslant k \leqslant n$, такой, что

$$
\gamma_{n}\left(H_{0}\right)\left(\xi_{k}\right)=(n-2 k) \xi_{k}, \quad \gamma_{n}\left(X_{0}\right)\left(\xi_{k}\right)=k(n-k+1) \xi_{k-1}, \quad \gamma_{n}\left(Y_{0}\right)\left(\xi_{k}\right)=\xi_{k+1}
$$

Следовательно, в базисе $\left(H_{0}, X_{0}, Y_{0}\right)$ ненулевьми элементами матрицы билинейной формы $B_{\gamma_{n}}$ являются

$$
\operatorname{Tr}\left(\gamma_{n}\left(H_{0}\right)^{2}\right)=\sum_{k=0}^{n}(n-2 k)^{2}=\frac{n(n+1)(n+2)}{3}
$$

и

$$
\operatorname{Tr}\left(\gamma_{n}\left(X_{0}\right) \gamma_{n}\left(Y_{0}\right)\right)=\sum_{k=0}^{n}(k+1)(n-k)=\frac{n(n+1)(n+2)}{6}=\left(\begin{array}{c}
n+2 \\
3
\end{array}\right)
$$

а сама матрица имеет вид

$$
B_{n}=\left(\begin{array}{c}
n+2 \\
3
\end{array}\right)\left(\begin{array}{lll}
2 & 0 & 0 \\
0 & 0 & 1 \\
0 & 1 & 0
\end{array}\right)
$$

Это доказьвает предложение 1.

Для доказательства теоремы 1 заметим, что присоединенное представление $\mathrm{ad} \cong \gamma_{2}$, последовательность чисел $c_{2}\left(\gamma_{n}\right)=\left(\begin{array}{c}n+2 \\ 3\end{array}\right), n=1,2,3, \ldots$, возрастает и первые несколько ее членов равны $1,4,10, \ldots$.

Отметим, что

$$
\operatorname{Tr}\left(\gamma\left(H_{0}\right)^{2}\right)=2 \operatorname{Tr}\left(\gamma\left(X_{0}\right) \gamma\left(Y_{0}\right)\right)
$$

для всех представлений $\mathrm{sl}(2, \mathbb{C})$, поскольку это верно для всех неприводимых представлений.

1.3. Доказательство предложения 1 в общем случае. Пусть $r \geqslant 3$. Рассмотрим подалгебру Картана $\mathfrak{h} \subset \operatorname{sl}(r, \mathbb{C})$, состоящую из диагональных матриц с нулевым следом. Матрицу $H \in \mathfrak{h}$ будем задавать диагональными элементами $\left(\lambda_{1}, \ldots, \lambda_{r}\right)$.

Линейные формы $\lambda_{i}-\lambda_{j}, i \neq j$, образуют систему корней $R \subset \mathfrak{h}^{*}$. Множество $S=\left\{\alpha_{1}, \ldots, \alpha_{r-1}\right\}$, где $\alpha_{i}=\lambda_{i}-\lambda_{i+1}$, является базисом в этой системе. Двойственньй базис в дуальной системе $R^{*} \subset \mathfrak{h}$ образуют матрицы $H_{i}$, имеющие только два ненулевых элемента: $\lambda_{i}=1$ и $\lambda_{i+1}=-1, i=1, \ldots, r-1$. Отметим, что $\alpha_{i}$ отождествляется с $H_{i}$ при изоморфизме $\mathfrak{h}^{*} \cong \mathfrak{h}$, которьй задается формой Киллинга

$$
(X ; Y)=\operatorname{Tr}(X Y)=\left.B_{\text {can }}\right|_{\mathfrak{h}}(X ; Y)
$$


Множество $R_{+}$положительных корней относительно $S$, состоящее из элементов вида $\alpha=\lambda_{i}-\lambda_{j}, i<j$, отождествляется при этом с множеством матрицвида $H_{\alpha}=H_{i}+\cdots+H_{j}$, также имеющих только два ненулевых элемента: $\lambda_{i}=1$ и $\lambda_{j}=-1$.

Рассмотрим стандартный базис в $\operatorname{sl}(r, \mathbb{C})$, состояший из матриц $H_{i}$ (описанных выше), $X_{\alpha}$ и $Y_{\alpha}, \alpha \in R_{+}$, где для $\alpha=\lambda_{i}-\lambda_{j}, i<j$, матрица $X_{\alpha}$ имеет единственньй ненулевой элемент 1 на месте $(i, j)$, и $Y_{\alpha}=X_{\alpha}^{T}$. Матрищы $H, X_{\alpha}$ и $Y_{\alpha}$ связаны соотношениями

$$
\left[X_{\alpha}, Y_{\alpha}\right]=H_{\alpha}, \quad\left[H, X_{\alpha}\right]=\alpha(H) X_{\alpha}, \quad\left[H, Y_{\alpha}\right]=-\alpha(H) Y_{\alpha} \quad \forall H \in \mathfrak{h} .
$$

Пусть $\Gamma$ - конечномерньй неприводимьй $\operatorname{sl}(r, \mathbb{C})$-модуль, $P$ - множество его весов. Для каждого $\pi \in P$ обозначим через $\Gamma^{\pi}$ соответствующее весовое подпространство, а число $m_{\pi}=\operatorname{dim} \Gamma^{\pi}$ назовем кратностью веса $\pi$. Известно, что $P$ инвариантно относительно действия групш Вейля $W$, порожденной отражениями относительно корней, и что для любых $\pi \in P$ и $w \in W$ кратности весов $\pi$ и $w(\pi)$ совпадают. Кроме того,

$$
\Gamma=\bigoplus_{\pi \in P} \Gamma^{\pi}
$$

Покажем, что элементы матрицы билинейной формы $\operatorname{Tr}(X Y)$, где $X$ и $Y$ понимаются уже как операторы на $\Gamma$, в указанном выше базисе удовлетворяют следующим соотношениям:

(i) $\operatorname{Tr}\left(X_{\alpha} Y_{\beta}\right)=0, \quad \alpha \neq \beta, \quad \operatorname{Tr}\left(H_{i} X_{\alpha}\right)=\operatorname{Tr}\left(H_{i} Y_{\alpha}\right)=0$;

(ii) $\operatorname{Tr}\left(H_{i}^{2}\right)=\operatorname{Tr}\left(H_{j}^{2}\right)=2 \operatorname{Tr}\left(X_{\alpha} Y_{\alpha}\right) \quad \forall i, j, \alpha$;

(iii) $\operatorname{Tr}\left(H_{i} H_{j}\right)= \begin{cases}-\frac{1}{2} \operatorname{Tr}\left(H_{i}^{2}\right), & \text { если } \alpha_{i}+\alpha_{j} \in R, \\ 0 & \text { в противном случае. }\end{cases}$

Это будет означать, что вся матрица задается одним ненулевьм элементом, например, $\operatorname{Tr}\left(H_{1}^{2}\right)$, и, следовательно, для всех неприводимых $\mathrm{sl}(r, \mathbb{C})$-модулей соответствующие матрицы пропорциональны. Справедливость предложения 1 будет следовать из полной приводимости конечномерных $\mathrm{sl}(r, \mathbb{C})$-модулей.

Несложная проверка показывает, что $X_{\alpha}\left(\Gamma^{\pi}\right) \subset \Gamma^{\pi+\alpha}, Y_{\alpha}\left(\Gamma^{\pi}\right) \subset \Gamma^{\pi-\alpha}$ и $H_{i}\left(\Gamma^{\pi}\right) \subset \Gamma^{\pi}$, откуда следует (i).

Оператор $H_{i}$ действует на подпространстве $\Gamma^{\pi}$ как гомотетия с коэффициентом $\pi\left(H_{i}\right)$, поэтому

$$
\operatorname{Tr}\left(\left(H_{i}\right)^{2}\right)=\sum_{\pi \in P} \pi\left(H_{i}\right)^{2} m_{\pi}
$$

Поскольку существует $w \in W$, переводящий $H_{i}$ в $H_{\alpha}$, мы имеем

$$
\operatorname{Tr}\left(\left(H_{\alpha}\right)^{2}\right)=\sum_{\pi \in P} \pi\left(H_{\alpha}\right)^{2} m_{\pi}=\sum_{\pi \in P} \pi\left(w\left(H_{i}\right)\right)^{2} m_{w(\pi)}=\operatorname{Tr}\left(\left(H_{i}\right)^{2}\right) .
$$

Далее, элементы $H_{\alpha}, X_{\alpha}$ и $Y_{\alpha}$ порождают подалгебру $\mathfrak{g}_{\alpha}$, изоморфную $\mathrm{sl}(2, \mathbb{C})$, причем соотношения между ними те же, что и между $H_{0}, X_{0}$ и $Y_{0}$. В силу формулы (4), примененной к $\mathfrak{g}_{\alpha}$-модулю $\Gamma$, имеем $\operatorname{Tr}\left(H_{\alpha}^{2}\right)=2 \operatorname{Tr}\left(X_{\alpha} Y_{\alpha}\right)$. Это доказывает (ii).

Покажем справедливость (iii). Пусть $\alpha_{i}+\alpha_{j}=\alpha \in R$, тогда по свойствам базиса системы корней $\alpha \in R_{+}$. Имеем $H_{i}+H_{j}=H_{\alpha}$, и из равенства

$$
\operatorname{Tr}\left(H_{\alpha}^{2}\right)=\operatorname{Tr}\left(\left(H_{i}+H_{j}\right)^{2}\right)=\operatorname{Tr}\left(H_{i}^{2}\right)=\operatorname{Tr}\left(H_{j}^{2}\right)
$$


легко вытекает требуемое. Пусть теперь $\alpha_{i}+\alpha_{j} \notin R$, тогда $\left(H_{i}, H_{j}\right)=0$, и отражение $s_{i}$ относительно $H_{i}$ оставляет $H_{j}$ неподвижньм, а у $H_{i}$ меняет знак. Оператор $H_{i} H_{j}$ действует на $\Gamma^{\pi}$ как гомотетия с коэффициентом $\pi\left(H_{i}\right) \pi\left(H_{j}\right)$, следовательно,

$$
\operatorname{Tr}\left(H_{i} H_{j}\right)=\sum_{\pi \in P} \pi\left(H_{i}\right) \pi\left(H_{j}\right) m_{\pi}=\sum_{\pi \in P} \pi\left(s_{i}\left(H_{i}\right)\right) \pi\left(s_{i}\left(H_{j}\right)\right) m_{s_{i}(\pi)}=-\operatorname{Tr}\left(H_{i} H_{j}\right),
$$

откуда $\operatorname{Tr}\left(H_{i} H_{j}\right)=0$. Это завершает доказательство предложения.

1.4. Доказательство теоремы 1 для $\mathrm{su}(3)$. Как известно [5], имеется взаимно однозначное соответствие между неприводимыми $\mathrm{sl}(r, \mathbb{C})$-модулями и их старшими весами. При этом, если $\lambda$ - старший вес неприводимого представления $\gamma$, то условие конечномерности $\gamma$ состоит в том, чтобы все значения $\lambda\left(H_{i}\right), i=1, \ldots, r-1$, были цельми неотрицательными числами. Это означает, что $\lambda=k_{1} \omega_{1}+\cdots+k_{r-1} \omega_{r-1}$, где $k_{i} \in \mathbb{Z}$, $k_{i} \geqslant 0$, и $\omega_{i}-\not$ фундаментальные веса, определяемые равенствами $\omega_{i}\left(H_{j}\right)=\delta_{i j}$. По определению $c_{2}(\mathrm{can})=1$ и, очевидно, что для канонического представления $\operatorname{Tr}\left(H_{1}^{2}\right)=2$. Следовательно, для представления $\gamma$ имеем

$$
c_{2}(\gamma)=\frac{1}{2} \operatorname{Tr}\left(\gamma\left(H_{1}\right)^{2}\right)=\frac{1}{2} \sum_{\pi \in P} \pi\left(H_{1}\right)^{2} m_{\pi} \geqslant \frac{1}{2} \sum_{\pi \in \lambda^{W}} \pi\left(H_{1}\right)^{2} .
$$

Здесь $\lambda^{W}$ - орбита старшего веса относительно действия группы Вейля, а $m_{\lambda}$ всегда равняется 1 . Если $\lambda=k_{1} \omega_{1}+k_{2} \omega_{2}-$ старший вес представления $\gamma$ алгебры $\mathrm{sl}(3, \mathbb{C})$, то последняя сумма может быть легко вычислена. Она равна $4\left(k_{1}^{2}+k_{2}^{2}\right)$ при $k_{1}, k_{2}>0$ и $2 k^{2}$, если одно из $k_{1}, k_{2}$ равно 0 , а другое равно $k$. Пусть $\gamma$ входит в набор представлений, на котором бета-функция неположительна, тогда $c_{2}(\mathrm{ad})=6 \geqslant c_{2}(\gamma)$, откуда в первом случае $k_{1}^{2}+k_{2}^{2} \leqslant 3$, т.е. $k_{1}=k_{2}=1$ и $\gamma=\mathrm{ad}$, а во втором-имеется четыре возможности:

\begin{tabular}{|c|c|c|c|c|}
\hline$\left(k_{1}, k_{2}\right)$ & $(1,0)$ & $(2,0)$ & $(0,1)$ & $(0,2)$ \\
\hline$\gamma$ & can & $S^{2}(\operatorname{can})$ & can $^{*}$ & $S^{2}\left(\right.$ can $\left.^{*}\right)$ \\
\hline$c_{2}(\gamma)$ & 1 & 5 & 1 & 5 \\
\hline
\end{tabular}

Это завершает доказательство.

1.5. Доказательство теоремы 1 для $\mathrm{su}(r)$. Утверждение а) очевидно, докажем б). В силу соотношений (5) имеем $\operatorname{ad} H_{1}\left(H_{i}\right)=\left[H_{1}, H_{i}\right]=0, \operatorname{ad} H_{1}\left(X_{\alpha}\right)=\left[H_{1}, X_{\alpha}\right]$ $=\alpha\left(H_{1}\right) X_{\alpha}$ и ad $H_{1}\left(Y_{\alpha}\right)=\left[H_{1}, Y_{\alpha}\right]=-\alpha\left(H_{1}\right) Y_{\alpha}$. Вычисляя след оператора $\left(\operatorname{ad} H_{1}\right)^{2}$, получаем требуемое

$$
c_{2}(\operatorname{ad})=\frac{1}{2} \operatorname{Tr}\left(\left(\operatorname{ad} H_{1}\right)^{2}\right)=2 r .
$$

Утверждение в) получается применением общей формулы (3). Фундаментальные веса имеют вид $\omega_{i}=\lambda_{1}+\cdots+\lambda_{i}, 1 \leqslant i \leqslant r-1$, причем $\left(\omega_{i} ; \omega_{k}\right)=i(r-k) / r$ при $i \leqslant k$. Полусумма положительных корней, как нетрудно видеть, равна

$$
\rho=\sum_{i=1}^{r-1} \omega_{i}
$$


Старшие веса представлений $\Lambda^{k}(\mathrm{can})$ и $S^{k}(\mathrm{can})$ равны соответственно $\omega_{k}$ и $k \omega_{1}$. Вычисляя коэффициенты гомотетии по формуле (2), имеем

$$
\left(\omega_{k} ; \omega_{k}+2 \rho\right)=\frac{k(r-k)(r+1)}{r} \quad \text { и } \quad\left(k \omega_{1} ; k \omega_{1}+2 \rho\right)=\frac{k(r+k)(r-1)}{r} .
$$

В силу (3), если умножить эти числа соответственно на

$$
\operatorname{dim} \Lambda^{k}(\operatorname{can})=\left(\begin{array}{l}
r \\
k
\end{array}\right) \quad \text { и } \quad \operatorname{dim} S^{k}(\operatorname{can})=\left(\begin{array}{c}
r+k-1 \\
k
\end{array}\right),
$$

а затем разделить на $r^{2}-1=\operatorname{dim} \operatorname{sl}(r, \mathbb{C})$, то получим

$$
c_{2}\left(\Lambda^{k}(\operatorname{can})\right)=\left(\begin{array}{l}
r-2 \\
k-1
\end{array}\right) \quad \text { и } \quad c_{2}\left(S^{k}(\operatorname{can})\right)=\left(\begin{array}{l}
r+k \\
k-1
\end{array}\right) .
$$

При $k=2$ из этих формул имеем $c_{2}\left(\Lambda^{k}(\mathrm{can})\right)=r-2$ и $c_{2}\left(S^{k}(\mathrm{can})\right)=r+2$, что ввиду $(6)$ и (1) завершает доказательство.

\section{§2. От полевых уравнений к алгебро-геометрическим проблемам}

Теорема 1 показывает, что у нас есть три серии - а), б) и в), - имеющие место для любой $\mathrm{su}(r)$. В этом параграфе мы рассмотрим первые две из них.

2.1. Для серии а) наши поля состоят из мультиплетов в присоединенном представлении. Мы пишем полевые уравнения для специального типа метрик, а именно, кэлеровых метрик, потому что наши вычисления будут относиться только к случаю алгебраических поверхностей, где нужные нам константы будут иметь алгебро-геометрический смысл и поэтому могут быть вычислены в рамках алгебраической геометрии. Для простоты мы будем рассматривать односвязньй случай. Итак, пусть $S$ - односвязная кэлерова поверхность и $K$ - ее канонический класс. Конфигурационным пространством будет прямое произведение

$$
\mathscr{A}(E) \times \Gamma(\operatorname{ad} E \otimes K),
$$

где $\mathscr{A}(E)$ - аффинное пространство $\mathrm{SU}(r)$-связностей в комплексном расслоении $E$ на $S$ ранга $r$ с $c_{1}=0$ и $c_{2}=k, \Gamma(\operatorname{ad} E \otimes K)$ - пространство полей Хиггса-Хитчина. Тогда для точки $(a, \phi) \in \mathscr{A}(E) \times \Gamma(\operatorname{ad} E \otimes K)$ полевые уравнения имеют вид

$$
F_{a}^{2,0}=F_{a}^{0,2}=0, \quad \bar{\partial}(\phi)=0, \quad \omega \wedge F_{a}+[\phi, \bar{\phi}]=0,
$$

где $F_{a}^{i, j}$ - компоненты разложения Ходжа тензора кривизны $F_{a}$ связности $a$ и $\omega-$ кэлерова форма нашей метрики. Используя линеаризацию этих уравнений, легко показать, что для общей метрики пространство решений ориентируемо и конечно, так что для любого $k=c_{2}(E)$ мы получаем число $\#_{k}$ этих решений с учетом знака. Наша статсумма в этом случае имеет вид

$$
Z_{(\mathrm{a})}=\sum_{k=0}^{\infty} \#_{k} q^{k}
$$

где, как обычно, $q=e^{2 \pi i k \tau}$ (подробности см. в [8]). 
Геометрический смысл уравнений (8) следующий: первое уравнение эквивалентно утверждению, что форма кривизны имеет тип $(1,1)$, что эквивалентно заданию голоморфной структуры на $E$; второе уравнение эквивалентно голоморфности поля Хиггса-Хитчина; и третье уравнение имеет смысл нулевого уровня отображения моментов для действия калибровочной группы, т.е. означает некоторые условия стабильности для голоморфной пары $(E, \phi: E \rightarrow E \otimes K)$.

Теперь предположим, что для нашей кэлеровой структуры на $S$ это условие стабильности влечет зануление голоморфного поля Хиггса-Хитчина $\phi$. Так будет, например, если канонический класс $K \leqslant 0$, т.е. если $S$ - поверхность дель Пеццо или типа К3. В этом случае наша кэлерова метрика не является общей, так как система уравнений (8) сводится к уравнению автодуальности на связность $a$ и многообразие модулей решений есть все пространство $\mathscr{M}_{k}(r) \mathrm{SU}(r)$-инстантонов на $S$ с зарядом $k$. Например, при $r=2$ это многообразие имеет размерность $4 k-3$ для поверхностей дель Пещцо и $4 k-6$ для поверхностей типа К3. Для регуляризации задачи мы должны применить деформацию к нормальному конусу, как это описано в работе [9, гл. III]. В этом случае расслоение препятствий на $\mathscr{M}_{k}(r)$ имеет слоем коядро оператора $\bar{\partial}$, т.е. для точки $E \in \mathscr{M}_{k}(r)$ слой расслоения препятствий есть $H^{1}(\operatorname{ad} E \otimes K)$ в силу изоморфизма Дольбо. Таким образом, расслоение препятствий для нашей задачи есть кокасательное расслоение $\Omega \mathscr{M}_{k}(r)$ в силу изоморфизма Кодаиры-Спенсера. Согласно конструкции, изложенной в работе $[9$, гл. III], число решений нашей задачи есть

$$
\#_{k}=c_{\mathrm{top}}\left(\Omega \mathscr{M}_{k}(r)\right)
$$

т.е. старший класс Чжэня кокасательного расслоения.

ЗАмЕчАниЕ. Многообразие $\mathscr{M}_{k}(r)$ может быть некомпактным (например, это имеет место для $r=2$ ), и мы должны его рассматривать как многообразие "с концами". Мы должны оценить, какое число решений уходит на бесконечность по этим "концам" от общего числа решений, задаваемого топологической эйлеровой характеристикой компактификации Фрид-Уленбек многообразия $\mathscr{M}_{k}(r)$ (см. [10]), рассматриваемой как орбифолд. (К сожалению, этот анализ отсутствует в основополагающей работе [2], и читателю приходится проделывать его самостоятельно, пользуясь, как образцом, аналогичной задачей о выражении спин-полиномов через полиномы Дональдсона,первый шаг решения которой описан в [11].)

Таким образом, (после реализации программы, изложенной в замечании) статсумма для серии а) имеет вид

$$
Z_{(\mathrm{a})}=\sum_{k=0}^{\infty} \chi\left(\overline{\mathscr{M}_{k}(r)}\right) q^{k}
$$

где $\chi$ - топологическая эйлерова характеристика $\overline{\mathscr{M}_{k}(r)}$ как орбифолда.

Тестирование этой суммы на модулярность, проведенное в [2] (для поверхностей типа $\mathrm{K} 3$ и $\mathbb{C P}^{2}$ ), основывается на замечательной работе А. Клячко [12], выразившего коэффициенты ряда (10) в терминах арифметической функции Гурвища.

2.2. Перейдем к серии б). K сожалению, этот случай не описан в литературе, и мы рассмотрим его подробно. 
Пусть $M, g$ - гладкое, компактное, односвязное риманово многообразие. Рассмотрим связность Леви-Чивита на касательном расслоении. Выбор Spin ${ }^{\mathbb{C}}$-структуры дает нам пару комплексных эрмитовых расслоений $W^{ \pm}$ранга 2 с классом $c=c_{1}\left(\operatorname{det} W^{ \pm}\right)$.

Связность Леви-Чивита индуцирует $\mathrm{SO}(3)$-связности на $W^{ \pm}$. Рассмотрим эрмитово расслоение $E$ ранга $r$ с унитарной связностью $a$, определенной с точностью до гомотетии. Кроме того, рассмотрим $U(1)$-связность $b$ на линейном $\operatorname{paccлоении~} \operatorname{det}\left(E \otimes W^{+}\right)$.

Конфигурационным пространством наших полевых уравнений будет множество наборов $\left(a, b, \phi_{1}, \ldots, \phi_{2 r}\right)$, где первый элемент - унитарная связность с точностью до гомотетии на $E$, второй - $U(1)$-связность $b$ на линейном $\operatorname{paccлоении~} \operatorname{det}\left(E \otimes W^{+}\right)$и $\phi_{i}-$ сечения тензорного произведения $E \otimes W^{+}$.

Напомним, что оператор Дирака $D_{a, b}$ - дифференциальньй оператор на пространствах сечений $E \otimes W^{+}$, задающийся следующим образом. Ковариантная производная задает отображение

$$
d_{a, b}: \Gamma\left(E \otimes W^{+}\right) \rightarrow \Gamma\left(E \otimes W^{+}\right) \otimes \Gamma\left(T^{*} M\right),
$$

и последнее тензорное произведение сворачивается как

$$
\Gamma\left(E \otimes W^{+} \otimes T^{*} M\right)=\Gamma\left(E \otimes W^{+} \otimes \operatorname{Hom}\left(W^{-}, W^{+}\right)^{*}\right)=\Gamma\left(E \otimes W^{-}\right)
$$

посредством клиффордова умножения. Композиция ковариантного дифференцирования (11) и свертки дает скрученный оператор Дирака

$$
D_{a, b}: \Gamma\left(E \otimes W^{+}\right) \rightarrow \Gamma\left(E \otimes W^{-}\right),
$$

и наши полевые уравнения имеют вид

$$
D_{a, b}\left(\phi_{i}\right)=0, \quad F_{a}^{+}=\sum_{i} i \cdot\left(\phi_{i} \otimes \overline{\phi_{i}}\right)_{0},
$$

где индекс 0 означает взятие бесследной части в $\operatorname{End}\left(W^{+}\right)$.

ЗАмЕчАнИЕ. Можно заметить, что эти уравнения имеют вид нулевого уровня отображения моментов гиперкэлеровой редукции относительно калибровочной групшы. В частности, первое уравнение содержит интерпретацию нулевого уровня отображения моментов для голоморфной симплектической структуры.

Теперь мы подбираем расслоение $E$ так, чтобы многообразие модулей $\mathscr{M}_{c}(E)$ орбит решений (13) было конечным. Пусть \# $\left(\mathscr{M}_{c}^{g}(E)\right)$ - число решений с учетом знака, т.е. ориентации. Зависимость \# $\left(\mathscr{M}_{c}^{g}(E)\right)$ от расслоения $E-$ это зависимость от топологического типа этого расслоения. То есть мы можем записать

$$
\#\left(\mathscr{M}_{c}^{g}(E)\right)=\#\left(\mathscr{M}_{c}^{g}\left(r, c_{1}, k\right)\right)
$$

где, как обычно, $k=c_{2}(E)$.

Наша статсумма в этом случае имеет вид

$$
Z_{(б)}=\sum_{k=0}^{\infty} \#\left(\mathscr{M}_{c}^{g}\left(r, c_{1}, k\right)\right) q^{k}
$$

где опять $q=e^{2 \pi i k \tau}$ и метрика $g$ является достаточно общей (для необщих метрик наше многообразие модулей может иметь параметры). 
ЗАмЕчАниЕ. Нетрудно видеть, что наши уравнения задают мультиплет неабелевых монополей, обобщающих абелев монополь Зайберга-Виттена (см., например, [13]).

Напомним, что при описании серии а) мы сразу исходили из кэлеровой метрики, т.е. сводили задачу к чисто алгебро-геометрической проблеме. Чтобы сделать это же в серии б), мы должны действовать более аккуратно (см. следующий параграф). Здесь мы проиллюстрируем общую ситуацию на примере случая $r=2$ и $M=\mathbb{C P}^{2}$ - топологически простейшей алгебраической поверхности.

Рассмотрим в качестве $g$ стандартную метрику $g_{\mathrm{FS}}$ Фубини-Штуди. Эта метрика имеет положительную скалярную кривизну. Формула Вейценбока показывает, что в этом случае мультиплет скрученных гармонических спиноров $\left(\phi_{1}, \ldots, \phi_{4}\right)$ должен быть равен $(0, \ldots, 0)$, и полевое уравнение сводится к уравнению автодуальности. Многообразие модулей решений $\mathscr{M}_{c}^{g_{\mathrm{FS}}}\left(2, c_{1}, k\right)$ совпадает с многообразием модулей инстантонов этого топологического типа. Арифметические вычисления следующего параграфа показывают, что единственная возможность для $c_{1}$ есть -1 и $\operatorname{dim} \mathscr{M}_{c}^{g_{\mathrm{FS}}}(2,-1, k)=4 k-4$.

Опять для регуляризации задачи мы должны применить деформацию к нормальному конусу, как это описано в работе [9, гл. III]. В этом случае расслоение препятствий на $\mathscr{M}_{c}^{g \mathrm{FS}}(2,-1, k)$ имеет слоем прямую сумму четырех коядер скрученного оператора Дирака (12). Как обычно, мы интерпретируем автодуальную связность как голоморфное стабильное расслоение $E$ и оператор Дирака как прямую сумму $\bar{\partial} \oplus \bar{\partial}^{*}$ (см., например, [10]), т.е. коядро оператора Дирака есть пространство когерентных когомологий $H^{1}(E)$.

Таким образом, наше многообразие модулей $\mathscr{M}_{c}^{g \mathrm{FS}}(2,-1, k)$ есть многообразие модулей стабильных пучков без кручения данного топологического типа (следовательно, это многообразие компактно и гладко) и для точки $E \in \mathscr{M}_{c}^{g_{\mathrm{FS}}}(2,-1, k)$ слой расслоения препятствий есть $H^{1}(E)^{\oplus 4}$. Согласно конструкции, изложенной в работе [9, гл. III], число решений нашей задачи есть

$$
\#\left(\mathscr{M}_{c}^{g}(2,-1, k)\right)=c_{\text {top }}\left(\left(\mathscr{H}^{1}\right)^{\oplus 4}\right)
$$

где $\mathscr{H}^{1}$ - расслоение на многообразии модулей со слоем $H^{1}(E)$ и специально выбранной нормировкой (см. [6]).

В отличие от серии а) (см. замечание после формулы (9)) эта абсолютно точно поставленная задача успешно решается А. Л. Городенцевым и М.И. Леенсоном.

\section{§ 3. Арифметика серии б)}

Так же, как и в примере в конще предыдущего параграфа, мы интерпретируем многообразие модулей $\mathscr{M}_{c}^{g}\left(r, c_{1}, k\right)$ решений полевых уравнений как многообразие модулей стабильных пучков $E$ без кручения данного топологического типа. Расслоение препятствий имеет слоем прямую сумму $2 r$ коядер скрученного оператора Дирака (12), т.е. $H^{1}(E)^{\oplus 2 r}$. Следовательно, чтобы наша задача имела конечное число решений, необходимо и достаточно равенство размерностей

$$
\operatorname{dim} \mathscr{M}_{c}^{g}\left(r, c_{1}, k\right)=2 r \operatorname{dim} H^{1}(E) .
$$

Для поверхностей дель Пещцо это условие при помощи теоремы Римана-Роха может быть записано в терминах дискретных инвариантов $\left(r, c_{1}, k\right)$. Пусть $S$ - поверхность 
дель Пеццо и $K$ - ее канонический класс. Стабильность пучка $E$ понимается в смысле Мамфорда-Такемото относительно $(-K)$, т.е. $E$ не должен иметь подпучков меньшего ранга и большего или равного наклона $\mu=c_{1} \cdot(-K) / r$. Основньгм нашим ограничением на топологические инварианты пучка является неравенство

$$
-K^{2}<\mu(E)<0
$$

Если оно выполнено, то из свойств стабильности и двойственности Серра вытекает, что $H^{0}(E)=H^{2}(E)=0$ и

$$
\operatorname{dim} H^{1}(E)=-\chi(E)=k-\frac{1}{2} c_{1} \cdot\left(c_{1}-K\right)-r .
$$

Далее, пространство $\operatorname{Ext}^{1}(E, E)$ является формальньм касательным пространством $\mathrm{k}$ $\mathscr{M}_{c}^{g}\left(r, c_{1}, k\right)$ в точке $E$. Из стабильности пучка $E$ следует его простота, что эквивалентно $\operatorname{dim} \operatorname{Hom}(E, E)=1$. Наконец, $\operatorname{dim} \operatorname{Ext}^{2}(E, E)<\operatorname{dim} \operatorname{Hom}(E, E)$ в силу двойственности Серра и обильности $(-K)($ см. $[14])$, поэтому $\operatorname{Ext}^{2}(E, E)=0$. Таким образом,

$$
\operatorname{dim} \mathscr{M}_{c}^{g}\left(r, c_{1}, k\right)=-\chi(E, E)=-\chi\left(E^{*} \otimes E\right)=2 r k-(r-1) c_{1}^{2}-r^{2}+1 .
$$

Учитьвая два последних равенства, (14) принимает вид

$$
c_{1}^{2}-r c_{1} \cdot K+r^{2}+1=0 .
$$

Особенно интересные решения этого уравнения получаются на комплексной проективной плоскости.

ТЕОремА 2. Стабильный пучок $E$ на $\mathbb{P}^{2}$, дискретные инварианты которого удовлетворяют неравенству $-3<c_{1} / r<0$, обладает свойством (14) тогда $u$ только тогда, когда тройка чисел $\left(r,-c_{1}, 1\right)$ является решением уравнения Маркова

$$
x^{2}+y^{2}+z^{2}=3 x y z
$$

ДокАЗАТЕЛьСтво. Для $\mathbb{P}^{2}$, очевидным образом, $c_{1} \cdot K=-3 c_{1}$, откуда легко вытекает требуемое ввиду вьшеприведенных рассуждений.

Таким образом, числа $r$ и $-c_{1}$ являются числами Маркова из “одной ветви дерева Маркова". Выпишем несколько пар $\left(r, c_{1}\right)$ в предположении, что $r>-c_{1}$ :

$$
(2,-1),(5,-2),(13,-5),(34,-13),(89,-34),(233,-89), \ldots \text {. }
$$

Пара, следующая за $\left(r, c_{1}\right)$ в этой последовательности, имеет вид $\left(3 r+c_{1},-r\right)$, что согласуется с перестройками решений уравнения (18), состоящими в переходе ко второму корню квадратного уравнения по одной из переменных (в нашем случае $\left.\left(-c_{1}\right)\right)$ при фиксированных двух остальньг (см., например, [15]). Как известно [15], ранги исключительных расслоений на $\mathbb{P}^{2}$, составляющих виток спирали, образуют решение уравнения (18). И наоборот, для любого решения этого уравнения существует бесконечно много (за счет подкрутки) витков с такими рангами расслоений. При этом перестройки спиралей согласуются с перестройками решений уравнения (18). Множество решений с фиксированным значением 1 третьей переменной, полученное в теореме 2 , соответствует множеству витков вида $\left(E, F, \mathscr{O}_{\mathbb{P}^{2}}\right)$. 
ЗАмЕЧАнИЕ. Наблюдаемая здесь связь с исключительными расслоениями, с одной стороны, является основньм поводом для публикации этой статьи, a, с другой стороны, придает теории исключительных расслоений новьй геометрический смысл.

Рассмотрим теперь в качестве нашей поверхности квадрику $\mathbb{P}^{1} \times \mathbb{P}^{1}$. Еегруппа Пикара стандартньм образом отождествляется с $\mathbb{Z} \oplus \mathbb{Z}$, в соответствии с этим мы полагаем $c_{1}(E)=(a, b)$. Уравнение $(17)$ переписьвается в виде

$$
r^{2}+2 r(a+b)+2 a b+1=0 .
$$

Отсюда видно, что $r$ обязательно нечетно (отметим, что ранги исключительных расслоений на $\mathbb{P}^{1} \times \mathbb{P}^{1}$ также нечетны). Более того, для любого нечетного $r>0$ существуют решения вида

$$
(r,(a, b))=(1-2 a,(a,-1)), \quad \text { где } a=0,-1,-2, \ldots
$$

Наклон расслоения $E$ с такими рангом и $c_{1}$ равен $2(a-1) /(1-2 a)$, он удовлетворяет условию $-2 \leqslant \mu(E)<-1$, в то время как $K^{2}=8$, т.е. неравенство (15) вьполнено. Следовательно, справедливо также и условие на размерности (14).

Приведенное выше множество решений является ни чем иньм как подмножеством множества целых точек, лежащих на одной из прямолинейных образующих однополостного гиперболоида. Действительно, уравнение (19) равносильно уравнению $(r+a+b)^{2}-a^{2}-b^{2}+1=0$. Сделав стандартные преобразования

$$
(r+a+b)^{2}-a^{2}=b^{2}-1 \Longleftrightarrow(r+b)(r+2 a+b)=(b-1)(b+1),
$$

получаем уравнения двух семейств образующих гиперболоида

$$
\left\{\begin{array} { r l } 
{ k _ { 1 } ( r + b ) } & { = l _ { 1 } ( b - 1 ) , } \\
{ l _ { 1 } ( r + 2 a + b ) } & { = k _ { 1 } ( b + 1 ) , }
\end{array} \quad \left\{\begin{array}{r}
k_{2}(r+b)=l_{2}(b+1) \\
l_{2}(r+2 a+b)=k_{2}(b-1)
\end{array}\right.\right.
$$

Здесь $\left[k_{i}: l_{i}\right] \in \mathbb{R P}^{1}$ - параметры семейств. Зафиксировав один из них (разумеется, с рациональным отношением $\left[k_{i}: l_{i}\right]$ ), легко получить все точки с целыми координатами на соответствующей прямой стандартными средствами аналитической геометрии. В частности, решения (20) лежат на прямой из второго семейства, имеющей $\left[k_{2}: l_{2}\right]=[0: 1]$. В качестве примера приведем еще одну серию целочисленных решений уравнения (19), для которых $r>0$,

$$
(r,(a, b))=(3+4 t,(-7-12 t,-4-5 t)), \quad \text { где } t=0,1,2, \ldots,
$$

лежащую на прямой первого семейства с $\left[k_{1}: l_{1}\right]=[5: 1]$. Начальной точкой этой серии является $(3,(-7,-4))$. Многообразие модулей полустабильных пучков $E$ на $\mathbb{P}^{1} \times \mathbb{P}^{1} \mathrm{c}$ такими инвариантами $\left(r, c_{1}\right)$ и $c_{2} \geqslant 20$ исследовано в работе [7] именно в связи с тем, что для $E$ справедливо (14). Нетрудно убедиться, что и для пучков $E$, имеющих в качестве ранга и $c_{1}$ остальные решения из этой серии, вьполняется (15) и, следовательно, и (14). 


\section{§4. Алгебро-геометрическая модель для серии в)}

Здесь мы предполагаем, что $E$ - стабильное расслоение ранга $r \geqslant 3$ на поверхности дель Пеццо и

$$
-\frac{1}{2} K^{2}<\mu(E)<0 .
$$

Пространства $H^{0}\left(S^{2} E\right)$ и $H^{0}\left(\Lambda^{2} E\right)$ естественно отождествляются с пространствами соответственно симметрических и кососимметрических морфизмов расслоений $E^{*} \rightarrow E$. Но из свойств стабильности и неравенства $\mu(E)<0<\mu\left(E^{*}\right)$ следует, что $\operatorname{Hom}\left(E^{*}, E\right)=0$. Поэтому и $H^{0}\left(S^{2} E\right)=H^{0}\left(\Lambda^{2} E\right)=0$.

В силу двойственности Серра $H^{2}\left(S^{2} E\right)^{*}=H^{0}\left(S^{2} E^{*} \otimes K\right)$, причем последнее пространство естественно отождествляется с пространством морфизмов расслоений $\varphi: E \rightarrow E^{*} \otimes K$, обладающих свойством $\varphi^{*}(K)=\varphi$. Поскольку $0<\mu\left(E^{*}\right)<K^{2} / 2$, имеем $\mu\left(E^{*} \otimes K\right)<-K^{2} / 2<\mu(E)$, откуда следует, что $\operatorname{Hom}\left(E, E^{*} \otimes K\right)=0$, и, таким образом, $H^{2}\left(S^{2} E\right)=0$. По аналогичным соображениям и $H^{2}\left(\Lambda^{2} E\right)=0$. Следовательно, размерности пространств $H^{1}$ от симметрического и внешнего квадрата расслоения $E$ могут быть выражены через дискретные инварианты $\left(r, c_{1}, k\right)$ по теореме Римана-Роха:

$$
\begin{aligned}
& \operatorname{dim} H^{1}\left(S^{2} E\right)=-\chi\left(S^{2} E\right)=(r+2) k-\frac{r+3}{2} c_{1}^{2}+\frac{r+1}{2} c_{1} \cdot K-\frac{r(r+1)}{2}, \\
& \operatorname{dim} H^{1}\left(\Lambda^{2} E\right)=-\chi\left(\Lambda^{2} E\right)=(r-2) k-\frac{r-1}{2} c_{1}^{2}+\frac{r-1}{2} c_{1} \cdot K-\frac{r(r-1)}{2} .
\end{aligned}
$$

Принимая во внимание (16), заключаем, что необходимое условие на размерности

$$
\operatorname{dim} \mathscr{M}\left(r, c_{1}, k\right)=\operatorname{dim} H^{1}\left(S^{2} E\right)+\operatorname{dim} H^{1}\left(\Lambda^{2} E\right)
$$

эквивалентно уравнению на $r$ и $c_{1}$ :

$$
2 c_{1}^{2}-r c_{1} \cdot K+1=0
$$

Легко убедиться, что на $\mathbb{P}^{2}$ это уравнение не имеет решений. Действительно, в этом случае $c_{1} \cdot K=-3 c_{1}$, и дискриминант (23) относительно $c_{1}$ равен $9 r^{2}-8$. Это не может быть квадратом целого числа при $r \geqslant 3$, так как разность между соседними квадратами $9 r^{2}-(3 r-1)^{2}=6 r-1>16$.

На $\mathbb{P}^{1} \times \mathbb{P}^{1}$ решений также нет в силу того, что $c_{1} \cdot K$ в этом случае четно.

Рассмотрим теперь поверхность $X_{m}$ - плоскость с $m$ раздутьми точками общего положения, где $1 \leqslant m \leqslant 8$. Имеем

$$
\operatorname{Pic} X_{m}=\mathbb{Z} \ell_{0} \oplus \mathbb{Z} \ell_{1} \oplus \mathbb{Z} \ell_{2} \oplus \cdots \oplus \mathbb{Z} \ell_{m}
$$

где $\ell_{0}$ - прообраз прямой, поднятой с $\mathbb{P}^{2}$, и $\ell_{i}, 1 \leqslant i \leqslant m,-$ исключительные кривые, являющиеся прообразами раздуваемых точек. Канонический класс поверхности $X_{m}$ имеет вид

$$
K=-3 \ell_{0}+\sum_{i=1}^{m} \ell_{i}
$$


и форма пересечения задается соотношениями

$$
\ell_{0}^{2}=1, \quad \ell_{i}^{2}=-1, \quad 1 \leqslant i \leqslant m ; \quad \ell_{i} \cdot \ell_{j}=0, \quad i \neq j
$$

Обозначив

$$
c_{1}=a \ell_{0}+\sum_{i=1}^{m} b_{i} \ell_{i}
$$

имеем

$$
c_{1} \cdot K=-3 a-\sum_{i=1}^{m} b_{i}, \quad c_{1}^{2}=a^{2}-\sum_{i=1}^{m} b_{i}^{2} .
$$

Пусть $c_{1} \cdot K=A$, тогда из $(23)$ находим $c_{1}^{2}=(r A-1) / 2$, и вместе с $(21)$ это дает систему условий

$$
\left\{\begin{array}{l}
\sum_{i=1}^{m} b_{i}+3 a+A=0, \\
\sum_{i=1}^{m} b_{i}^{2}=a^{2}-\frac{1}{2}(r A-1),
\end{array}\right.
$$

Первое уравнение задает гиперплоскость в евклидовом пространстве с координатами $\left(b_{1}, \ldots, b_{m}\right)$, а второе - сферу с центром в начале координат. Покажем, что во всех случаях, кроме одного, расстояние $d=|3 a+A| / \sqrt{m}$ от начала координат до гиперплоскости больше радиуса $R$ сферы. Рассмотрим

$$
m\left(d^{2}-R^{2}\right)=(9-m) a^{2}+6 a+A^{2}+\frac{m}{2}(r A-1) .
$$

Относительно $а$ это квадратный трехчлен с дискриминантом

$$
D=\frac{m}{2}(r A-1)\left(m-9+\frac{2 A^{2}}{r A-1}\right) .
$$

При $0<m<9-2 A^{2} /(r A-1)$ дискриминант отрицательный, поэтому всегда $d^{2}-R^{2}>0$, и система (24) решений не имеет. Следовательно, если решения есть, то число раздутых точек и $c_{1} \cdot K$ связаны соотношением $m \geqslant 9-2 A^{2} /(r A-1)$. С другой стороны, из третьего неравенства системы $(24)$ следует, что $m<9-2 A / r$, т.е. $m$ лежит в числовом полуинтервале

$$
I=\left[9-\frac{2 A^{2}}{r A-1} ; 9-\frac{2 A}{r}\right) .
$$

Поскольку $A$ и $r$ целые, то правые концы $I$ либо целые, либо отстоят от целых чисел не менее, чем на $1 / r$, в то время как длина $I$ равна

$$
\frac{2 A^{2}}{r A-1}-\frac{2 A}{r}=\frac{2}{r\left(r-\frac{1}{A}\right)} \leqslant \frac{2}{r(r-1)},
$$

что не превосходит $2 /(3 r)$ при $r \geqslant 4$. Таким образом, при $r \geqslant 4$ промежуток $I$ не содержит целых чисел, и система (24) решений не имеет. При $r=3$ непосредственным 
подсчетом убеждаемся, что имеется единственное решение: $m=8, A=1, a=-3$, $b_{1}=\cdots=b_{8}=1$. Итак, доказано

ПРЕДЛОЖЕНИЕ 2. СТабильный пучок Е ранга 3 на плоскости с $m$ раздутыми точками удовлетворяет условиям (21) и (22) тогда и только тогда, когда $m=8$ $u c_{1}(E)=K$.

Отметим, что ввиду взаимной простоты $c_{1}(E) \cdot K$ и $r(E)$ многообразие модулей стабильных пучков с данными инвариантами компактно и гладко, следовательно, мы имеем корректно поставленную алгебро-геометрическую задачу для проверки гипотезы $S$-двойственности. А именно, пусть $\mathscr{S}_{k}$ и $\mathscr{L}_{k}$ - расслоения на $\mathscr{M}(3, K, k)$ со слоем соответственно $H^{1}\left(S^{2} E\right)$ и $H^{1}\left(\Lambda^{2} E\right)$. Задача состоит в тестировании на модулярность функции

$$
\sum_{k} c_{\text {top }}\left(\mathscr{S}_{k} \oplus \mathscr{L}_{k}\right) q^{k}
$$

Московский институт электроники и математики

Поступило

07.10 .96

\section{СПИСОК ЦИТИРОВАННОЙ ЛИТЕРАТУРЫ}

[1] Montonen C., Olive D. Magnetic monopoles as gauge particle // Phys. Lett. 72B. 1977. P. 117-132.

[2] Vafa G., Witten E. A strong coupling test of $S$-duality // Nuclear Phys. B. 1995. V. 431. P. 3-77.

[3] Argyres Ph. C., Plesser M.R., Seiberg N. The moduli space of Vacua of $N=2$ SUSY QCD and duality in $N=2$ SUSY QCD // Nuclear Phys. B. 1996. V. 471. P. 159-194.

[4] Donagi R., Witten E. Supersymmetric Yang-Mills theory and integrable systems // Nuclear Phys. B. 1996. V. 460. P. 299-334.

[5] Fulton W., Harris J. Representation Theory. 1st Course. Berlin: Springer Verlag, 1990.

[6] Gorodentsev A. L., Leenson M. I. How to calculate the correlation function in twisted SYM $N=2, N_{f}=4$ QFT on projective plane. Preprint № 96-49. Bohn: Max-Planck-Institut für Mathematik, 1996.

[7] Kuleshov S. A. Moduli Space of Bundles on a Quadric. Preprint № 18: Warwick, 1996.

[8] Witten E. Monopoles and four-manifolds // Math. Res. Letts. 1994. V. 1. P. 769-796.

[9] Пидстригач В., Тюрин А. Н. Инварианты гладкой структуры алгебраической поверхности, задаваемые оператором Дирака // Изв. РАН. Сер. матем. 1992. Т. 52. № 2. С. 279-371.

[10] Donaldson S., Kronheimer P. The Geometry of Four-Manifolds. Oxford: Clarendon Press, 1990.

[11] Leung W.-M.R. On Spin ${ }^{\mathbb{C}}$-Invariants of Four-Manifolds. Thesis Ph.D. Oxford: Magdalen College, 1995.

[12] Клячко А. А. Модули векторных расслоений и числа классов // Функцион. анализ и его прилож. 1991. Т. 25. №1. С. 81-83.

[13] Тюрин Н.А. Необходимое и достаточное условие деформации $B$-монополя в инстантон // Изв. РАН. Сер. матем. 1996. Т. 60. № 1. С. 211-224.

[14] Кулешов С.А., Орлов Д. О. Исключительные пучки на поверхностях дель Пеццо // Изв. РАН. Сер. матем. 1994. Т. 58. № 3. С. 53-87.

[15] Gorodentsev A. L., Rudakov A. N. Exceptional vector bundles on projective spaces // Duke Math. J. 1987. V. 54. P. 115-130. 